\title{
What is a bioactive compound? A combined definition for a preliminary consensus
}

\author{
Abdelkarim GUAADAOUI ${ }^{1}$, Soumaya BENAICHA ${ }^{1}$, Naima ELMAJDOUB ${ }^{1}$, \\ Mohammed BELLAOUI ${ }^{2}$, Abdellah HAMAL ${ }^{1}$

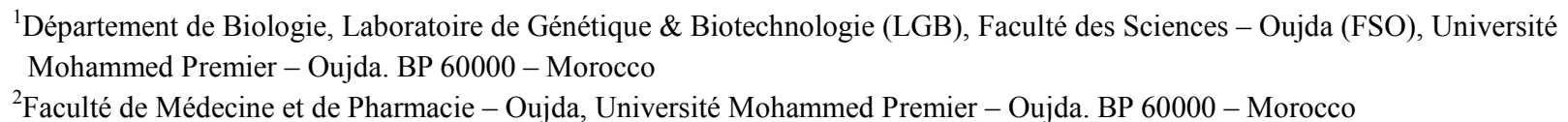

Email address:

abdelkarim119@gmail.com (A. GUAADAOUI)

\section{To cite this article:}

Abdelkarim GUAADAOUI, Soumaya BENAICHA, Naima ELMAJDOUB, Mohammed BELLAOUI, Abdellah HAMAL. What is a Bioactive Compound? A Combined Definition for a Preliminary Consensus. International Journal of Nutrition and Food Sciences. Vol. 3, No. 3, 2014, pp. 174-179. doi: 10.11648/j.ijnfs.20140303.16

\begin{abstract}
The definition of "bioactive compound" knowdynamism, because the scientific research on the bioactivity potential is booming nowadays. And since there is no unifying definition, attempts to find a consensus and some common elements of the concept associated with the term "bioactive" did not stop, even if vague definitions and repeated use of the operative word "bioactive" almost integrated the term in a category of magic words! This paper tried to find a combined definition which can be a platform to develop the "bioactive" concept, and reach a solid consensus on the term "bioactive compound". We use, for the first time, the term of "biocompounactive" tomore simplify the use of bioactive compound in relation with their physicochemical and biological properties.
\end{abstract}

Keywords: Bioactive Compound, Biocompounactive, Secondary Metabolites, Phytochemicals, Food Components, Healthy Diet

\section{Introduction}

Bioactive compounds are experiencing a growing interest in wide range of applications: geo-medicine, plant science, modern pharmacology, agrochemicals, cosmetics, food industry, nano-bio-science... etc. This is a very promising area in full development, which has resulted in research works more and more numerous, designed to diversify the resources of bioactive compounds and improve their salvage pathways or synthesis.

Despite all this significant number of research in different fields, the definition of bioactive compounds remains ambiguous and unclear. A very few references give a definition to the term "bioactive", and generally by a short sentence which does not leave the closed field of research, and thus limiting the generalization of these definitions.

This situation led us asking, for a long time, lots of questions, but first and foremost: What is a bioactive compound? To answer this question, we will, firstly, discuss different definitions collected, to arrive in a second time, to redraft a definition from the various concepts discussed.

\section{Bioactive Compound: Principles \& Concepts}

The term "bioactive" is composed by two words: bio- and -active. In etymology: bio- from the Greek (ßío-) "bios" [bio-, -bio], refers: life. And-active from the Latin "activus", means: dynamic, full of energy, with energy [1-3], or involves an activity [2]. This activity presents all the phenomena from which manifest a form of life, a functioning or a process [4].

In a strictly scientific sense, the term "bioactive" is an alternative term for "biologically active" [5]. A bioactive compound is simply a substance that has a biological activity [6].

In medical dictionaries, a bioactive substance is defined as a substance having an effect on [7], causes a reaction [8], or triggers a response in [9] the living tissue.

A compound(or a substance) having biological activity, if it has a direct effect on a living organism. These effects may be positive or negative depending on the substance, the dose or the bioavailability [10]. Indeed, these compounds have a 
wide range of effects, starting with the good maintenance of health even healing effect, or be dangerous even fatal. The ingested dose of bioactive compounds is often decisive for whether the effect positive or adverse[11].

Some authors consider that the demonstration of these activities alone is not sufficient for a compound will defined as "bioactive", it must also have an associated beneficial effect on health. This definition distinguishes these compounds from many others that are bioactive, but have adverse effects and are considered carcinogenic or toxic. This distinction affects the types of experiences that should be designed to investigate the bioactive compounds [12].

In addition to the effect of these compounds, another criterion involved in the definition of bioactive substances, is that of their origins. We can talk then of bioactive substances from food or other sources inedible by humans or animals.

In the first case, we speak about "non-nutritional uses of nutrients" [13], while exceeding the energy value of foods and their content of protein, fat and carbohydrates, in order to consider other ingredients that can contribute positively in the preservation of health through modification of normal physiological function or the improve of a biological activity of the organism [14].

Then, bioactive food components are components in foods or dietary supplements, other than those necessary to meet the basic nutritional needs, which are responsible for changes in health status [15]. In this case, it is important to understand that the bioactive compounds are not nutrients [12], even if they are contained in foods or their constituents.

These definitions opposes the view of Biesalski H-K et $a l$. (2009) who presented a definition in an envelope of 'consensus': "bioactive compounds" are essential and non-essential compounds that occur in nature, are part of the food chain, and can be shown to have an effect on human health [16].

Instead, several definitions exclude essential compounds from bioactive compounds. According to the Encyclopedia of Food \& Culture, the term "food bioactive component" refers to non-essential biomolecules that are present in foods and have the ability to modulate one or more metabolic processes, which results in the promotion of better health [17]. And close to the previous definition, "bioactive compounds" are:

- Extra-, non-nutritional components of food claimed to have beneficial health effects [18-19]; normally this does not include the essential nutrients [18].

- Extra-nutritional constituents that typically occur in small quantities in foods. They are extensively studied to evaluate their effects on health [20].

As already mentioned, the term bioactive food compound or components (bioactive dietary) is usually associated with only positive effects on the organism. Such definition considers that the bioactive dietary influence the state of health, and therefore have a biological value beyond their calorie content [10]. In this context, the bioactivity has the potential (at least) to affect health in a beneficial way, which excludes from the definition the potentially adverse effects (such as toxicity, allergenicity and mutagenicity) which are, undoubtedly, the reflection of "bioactivity" in its broadest sense [21].

In addition to this last declaration, the use of neutral sentences (health effects) or uncertain (provided, pretended, putative...) leaves a large margin to the "negative" effects of several compounds, on the living organism, to be included within the scope of "bioactivity". Thus, "bioactive compounds" can be defined as the inherent non-nutritive constituents of food plants with a provided beneficial promotion on health and/or toxic effects when ingested [22]. This approach takes a bigger presence in non-food compounds.

So far, it appears that several definitions attribute the term "bioactive compound" directly to compounds derived from foods (products or present in food, are part of the food chain...), in such a way, it is presented as synonymous with "bioactive food", and even more specifically limited to plants or food crops. While this is not the case!

For compounds derived from inedible sources, we can even imagine the "waste as a source of bioactive compounds"! [23, 65-67]. But, what interests us in this context, is the principles on which are based the definitions to understand the related concepts, considering here the criterion "origin" of these compounds, especially in organisms that produce them.

Bioactive compounds contain chemicals that are found in small quantities in plants [in general] and certain foods (such as fruits, vegetables, nuts, oils and whole grains); they have actions in the body that can promote good health [24].

In plants, nutrients are not generally included in the term "plant bioactive compound". Typical bioactive plant compounds are produced as secondary metabolites that are not necessary for the daily functioning of the plant (such as growth) [11], but play an important role in the competition, defense, attraction and signaling [25]. Bioactive compounds in the plants can be defined, then, as secondary plant metabolites eliciting pharmacological or toxicological effects in humans and animals [11].

Thus, plants are not the sole source of bioactive substances. These substances are also found in other living organisms and microorganisms, such as bacteria [26-30, 44] mushroom [19, 31-34, 44] and in some groups of animals [35-39]. What is said about the terrestrial (micro-)organisms, also applies to marine (micro-)organisms. These, produce too, potentially useful substances as bioactive secondary metabolites [29, 30, 35, 40-45].

It should be noted that in addition to natural bioactive substances $[46,47]$ the ability to synthesize a wide variety of bioactive molecules began in the early twentieth century, despite the development of pharmaceutical chemistry [48] and the emergence of new tools for chemical synthesis [49], thereby adding another source of bioactive molecules. It is synthetic source. 


\section{Bioactivity: Towards a Combined PreliminaryDefinition}

Identify bioactive compounds and establish their effects on health are active areas of scientific research [12], and as research on bioactivity potential is booming these days [50], this may explain, in part, the "dynamism"[51] that knows the definition of "bioactive" term, without neglecting the context of scientific studies that include different definitions.

And since there is no unifying definition [10], attempts to find a consensus and find some common elements of the concept associated with the term "bioactive" did not stop, even if vague definitions and repeated use of the operative word "bioactive" almost integrated the term in a category of "magic words" [50] !

Schrezenmeir J. et al.(2000) consider that the definition of bioactivity is usually refined by two caveats. One has already been discussed (positive and negative health effects), and the other requires in the bioactive component to give a measurable biological effect in a physiologically realistic level. Knowing that the range of physiological processes, that could be affected by this bioactivity, is vast [21]; which makes the accomplishment of this mission a difficult thing, and requires the intervention of several specialties and disciplines at the same time.

Answering the question: what is a bioactive compound? And redesigning a preliminary definition requires the combination of all concepts already mentioned, by replenishing the puzzle piece by piece to get a clear answer.

\subsection{Criterion 1:Essence and Origin}

\section{Is the Bioactivity Means that the Bioactive Compound is Necessarily"Bio"?}

Some definitions use terms like: biomolecules; occurs in nature... etc. But synthetic and semi-synthetic compounds are included in the concept of "bioactivity". Several products that are marketed (in cosmetics, medicine, agriculture...etc.), their active principles are based on synthetic molecules.

Generally, there is no difference in biological activity between the bioactive compounds derived from nature and synthetic products [52]. The example of coumarins illustrates the bioactivity of bioactive compounds, whether natural or synthetic origin [53-55]. So, in addition to the discoveries made by the isolation of coumarins from hundreds of species of plants and other organisms, there are synthetic derivatives that significantly increase the number of known structures of coumarin until today [56].

Indeed, the synthesis of coumarins and their derivatives has attracted considerable attention of organic and medicinal chemistry for many years because of potential applications in pharmaceuticals, cosmetics, and agrochemical industries [57]. Due to this incontestable importance of coumarin derivatives, and intensive use of these compounds in recent years, many strategies and analytical methods have been developed for the synthesis of coumarins [57, 58], and considerable efforts have been made by several researchers to prepare new compounds with simple substituents, or more complex systems, including heterocycles[59].

Therefore, a bioactive compound may be of natural or synthetic origin.

\section{Essential or Non-Essential? But what are?}

The term "bioactive compound" is not attributed to the nutrients contained in food or, more broadly, to the nutrients that are essential for a living organism, such as primary metabolites.

Controversies over except the essential elements of the definition of bioactive compounds arise for food (or sources of nutrition in general), with food constituents include water, carbohydrates, proteins, lipids and fatty acids, fibers, vitamins, minerals and oligo-element.

Some pre-mentioned definitions, consider that the bioactive peptides, many vitamins, fatty acids, flavonoids, phytosterols [6] and the soluble and insoluble fibers [17] are bioactive compounds. For Liu R.H. (2013), these bioactive compounds include phytochemicals, vitamins, minerals and fiber [60]. While other authors, such as NahlerG. (2013), for example, exclude vitamins and unsaturated fatty acids of this classification since they are essential nutrients [18].

To overcome this apparent contradiction, we give the example of proteins that have been increasingly recognized in recent years as physiologically active components in food and source of bioactive peptides [61].

According to present knowledge, bovine milk, cheeseand dairy products seem to be by far the greatestsources of bioactive proteins and peptides derivedfrom food[62].

Some food proteins cause specific effects beyond the supply of nutrients, such as IGFs (insulin-like peptide hormones), lactoferrin, immunoglobulins and $\beta$-lactoglobulin $(\beta-\mathrm{Lg})$.

The $\beta$ - $\mathrm{Lg}$ is now an important source of bioactive peptides that, once released, play an important role in human health, especially against hypertension, functionalities related to antioxidant and antimicrobial activities, and the ability to reduce levels of body cholesterol [63].

Bioactive peptides may be encrypted in the amino acid sequence of a larger protein. These peptides usually consist of 3-20 amino acids and are released from the original protein after degradation by hydrolysis or fermentation (Fig. 1)[62].

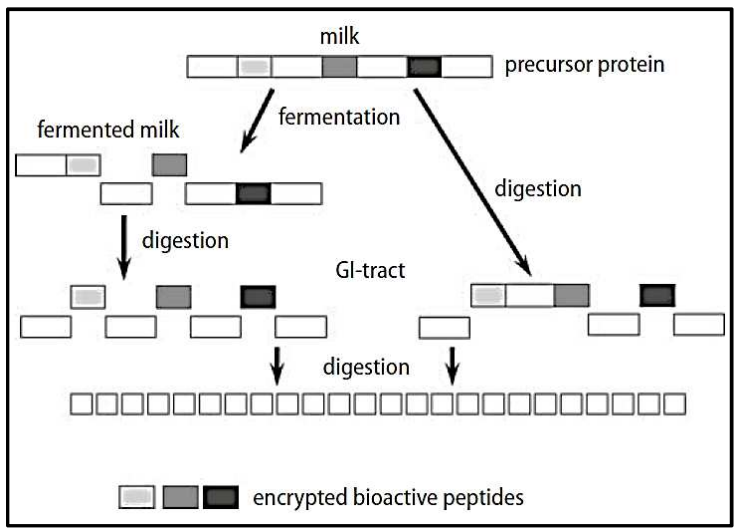

Figure 1. Scheme of peptides released from precursor proteins [62] 
It therefore appears that certain types of nutritional components can play a non- nutritional (or extra-nutritional) role, and present a bioactivity in the organism. But, once such compounds have biological activity, can we consider them as essential (nutritional role)?

In this case, we suggest that the bioactive compounds are non-essential, because the same compound (or molecule) cannot play two physiological roles at the same time in the same organism: one, nutritional (energetic metabolism and development), and other: bioactive (non-nutritional). This suggestion is aiming the distinction between two completely different processes: the first, which requires the degradation of the compound or molecule to release the essential energy for the functioning of the organism and its development, and the second, which requires the interaction of the compound (or molecule) in its integrality with one or more components of the living tissue.

Therefore, a bioactive compound is, too simply, a compound which has the capability to interact with one or more component(s) of the living tissue. So it comes back from itself and without need to mention it after, this interaction (bioactivity) can manifest whatever the source of bioactive compound: food and non-food, integrated into an essential nutrient or not. We can, in this case, be limited to refer to natural origin of the bioactive compound to define it, but with the evocation of the distinction suggested.

\subsection{Criterion 2: Bioactive Effect}

\section{Bioactivity: Positivity and/or Negativity?}

As already mentioned, some definitions bind the bioactivity just for the positive effects on the organism, and especially on human health, in such a way that this bioactivity will be beneficial for humans. But, searching different bioactive effects, including the "negative" effects, for human benefit was always, and will remain a large area of the scientist research, not only in terms of health, but also in various fields (agriculture, industry...), aspiring to improve the quality of human life at the end.

The range of effects that can exert a bioactive compound on different biological organisms is related to several factors, such as chemical structure, dose... etc. The change of one or more of these factors can make an expected negative effect positive, or the reverse. In addition, an effect which is positive for an organism in its entirety, or for a specific tissue of this organism, would not be for another, either directly or indirectly. The most spying examples that can be cited here, are those: (a) of the side effects (generally undesirable) which may be manifested within the same organism with the same bioactive compound, and (b) biocides, particularly those who are from natural origin, which would have, as appropriate, a positive effect on a living organism (or tissue) by fighting against another that it would be negative for it.

Therefore, a bioactive compound may present very different effects in one or more component(s) of living tissue; and this, according to several factors, which are usually linked to three major changes:

- The biocompounactive: The bioactive compound in relation with its physicochemical and biological properties (e.g. chemical structure, bioavailability...etc.);

- The subject involved in the biocompounactive use: the living organism in this case, and

- The manner of using the biocompounactive (dosage, alone or $\operatorname{mix}$... etc.).

\subsection{Criterion 3: the Quantity}

\section{The Small Quantity: Does become the Joker?}

From the above definitions, we find that some definitions include the criterion "quantity" in the definition of bioactive compounds:

- Components that usually occur in small amounts in foods [20];

- Bioactive compounds contain chemicals that are found in small amounts in some foods and plants [24];

- $\quad$ Minor components that have a potential impact on human health [64].

The integration of the criterion "quantity" in the definitions could be limiting to give a sense to the term "bioactive". But we must be very careful in this way!

\section{Conclusion}

\section{A return to the start...}

In conclusion, it is now clear that a bioactive compound is: "a compound which has the capability and the ability to interact with one or more component(s) of the living tissue by presenting a wide range of probable effects". The origin of these substances can be natural: terrestrial or aquatic; a plant, animal or other source (e.g. microorganisms) or synthetic: partially or totally. And why not, maybe one day an extraterrestrial source!

This combined definition is preliminary, and may constitute a platform to develop the concept of "bioactive", and specify, in addition, its scientific content in order to reach a solid consensus on the term, and avoid, consequently, the "chaotic" situation experienced by the term "bioactive compound" and its derivatives.

We used the term "biocompounactive" (in French: biocomposactif) for the first time, to more simplify the use of terms: bioactive compound and bioactive componentboth, in relation with their physicochemical and biological properties.

\section{Acknowledgements}

Abdelkarim G.thanks the Center of Doctoral Studies: Science and Technology (CEDoc-ST)-Oujda (Morocco), and all staff of the LGB-FSOlaboratory.

This work is the result of the efforts of all authors mentioned in the references. So a thanks for them all! 


\section{References}

[1] Bernard G and DromardA; Book of etymology and medical terminology: Lexicon etymology (in French). Livretd'étymologieet de terminologiemédicale: Lexiqued'étymologie, 2011: 1-4.

[2] Alain R ; Le Robert micro-poche (2nd Ed) (in french). 1994: 14 and 126

[3] Online Etymology Dictionary (website): http://www.etymonline.com; 2013.

[4] Anonymous ; Larousse de poche (in french), Les Editions Françaises Inc. $1994: 8$.

[5] Cammack R et al.; Oxford Dictionary of Biochemistry and Molecular Biology (2nd Ed). Oxford University Press, 2006: $74-75$.

[6] Dictionary of Food Science and Technology (2nd Ed). International Food Information Service (IFIS Editor), 2009: $47-48$.

[7] The American Heritage Medical Dictionary. Houghton Mifflin Company, 2007: 47.

[8] Mosby's Dictionary of Medicine, Nursing and Health Professions (9th Ed). Mosby Creator, 2013: 83.

[9] Miller-Keane and Marie TO; Miller-Keane Encyclopedia and Dictionary of Medicine, Nursing and Allied Health (7th Ed). Saunders, 2005: 62.

[10] Bioactive compounds, Food Bioactives Centre, University of Gothenburg http://www.chalmers.se/chem/fbac-en2012.

(website):

[11] AkselB; A brief review on bioactive compounds in plants. In: Bioactive compounds in plants - benefits and risks for man and animals. Oslo: The Norwegian Academy of Science and Letters, 2010: 11-17.

[12] Kris-Etherton PM and al.;Bioactive compounds in nutrition and health-research methodologies for establishing biological function: the antioxidant and anti-inflammatory effects of flavonoids on atherosclerosis. Annual Review of Nutrition, 2004, 24: 511-538.

[13] Richard JW; Non-nutritional uses of nutrients. European Journal of Pharmacology, 2011, 668(1): S10-S15.

[14] Guidelines for use of nutrition and health claims, CAC/GL 23-1997, Food and Agriculture Organization of the United Nations/ World Health Organization, Adopted in 1997. Revised in 2004. Amended in 2012: 2.

[15] Studdert VP et al.; Saunders Comprehensive Veterinary Dictionary (4th Ed). Elsevier Health Sciences UK, 2011: 79.

[16] Biesalski HK et al.; Bioactive compounds: Definition and assessment of activity. Nutrition, 2009, 25(11-12): 1202-1205.

[17] Solomon HK and William WW; Bioactive Food Components, Encyclopedia of Food \& Culture (2nd Ed). Acceptance to Food Politics, B Letter, Charles Scribner's Sons, 2003, 1: 201.

[18] NahlerG; Dictionary of Pharmaceutical Medicine (3rd Ed), B letter, Springer-Verlag Wien, 2013: 19-28.
[19] New data on bioactive compounds with putative health benefit (website): http://www.eurofir.net/publications/bioactive compounds20 11

[20] Kris-Etherton PM and al.;Bioactive compounds in foods: their role in the prevention of cardiovascular disease and cancer. American Journal of Medicine. 2002, 113(Suppl 9B): $71 \mathrm{~S}-88 \mathrm{~S}$.

[21] Schrezenmeir J et al.; Foreword. British Journal of Nutrition, 2000, 84(S1): 1.

[22] Gry J et al.;EuroFIR-BASIS - a combined composition and biological activity database for bioactive compounds in plant-based foods. Trends in Food Science \& Technology, 2007, 18(8): 434-444.

[23] Anonymous; Waste: A source of bioactive compounds. Biotechnology Journal, 2008, 3(7) : 1.

[24] NCI Dictionary of Cancer Terms, National Cancer Institute, Letter B, (website): http://www.cancer.gov/dictionary?cdrid=7032782013.

[25] Dudareva N and PicherskyE; Biochemical and molecular genetic aspects of floral scent. Plant Physiology, 2000, 122(3): 627-633.

[26] KarlovskyP; Secondary Metabolites in Soil Ecology, Part II Bacterial Metabolites, Springer, 2008: 93-127.

[27] ReichenbachH;Myxobacteria, producers of novel bioactive substances. Journal of industrial microbiology \& biotechnology, 2001, 27(3) : 149-156.

[28] Xie Y and al.; Bioactive natural products from Lysobacter. Natural product reports, 2012, 29(11): 1277.

[29] Rojas JL and al.; Bacterial diversity from benthic mats of Antarctic lakes as a source of new bioactive metabolites. Marine Genomics, 2009, 2(1): 33-41.

[30] Jaiganesh R and Sampath-Kumar NS; Marine bacterial sources of bioactive compounds. Advances in food and nutrition research, 2012, 65: 389.

[31] Zhou LL et al.; Effects on animal models of depression of bioactive compounds from entomogenous fungi, a novel antioxidant. Chinese Journal of Integrative Medicine, 2004, 10(3): $221-225$

[32] Debbab A et al.; Bioactive secondary metabolites from endophytes and associated marine derived fungi. Fungal Diversity, 2011, 49(1): 1-12.

[33] Peter CKC; Antitumor and Immunomodulatory Activities of Mushroom Polysaccharides: Mushrooms as Functional Foods. 2009: 147-198.

[34] Liu JK; Bioactive Secondary Metabolites from Higher Fungi in China. PlantaMedica, 2010, 76(5): S17.

[35] Suarez-Jimenez and al.;Bioactive peptides and depsipeptides with anticancer potential: sources from marine animals. Marine drugs, 2012, 10(5): 963-986.

[36] Clare DA and SwaisgoodHE;Bioactive milk peptides: a prospectus. Journal of dairy science, 2000, 83(6): 1187.

[37] Keizo A and MotokoO; Bioactive Compounds in Meat (Chapter 11). Meat Biotechnology, Springer, 2008: 231-249. 
[38] Rainer H et al.; Bioactive Egg Compounds. Springer-Verlag Berlin Heidelberg, 2007: 309p.

[39] Anne P and Hannu K; Bioactive Peptides and Proteins. Advances in food and nutrition research, 2003, 47: 175- 276.

[40] John JB and al.; Nature's bounty-drug discovery from the sea. Expert Opinion on Drug Discovery, 2007, 2(11): 1505-22.

[41] Alphonse K; Secondary metabolites from marine microorganisms, Anais da Academia Brasileira de Ciências, 2002, 74(1): 151-170.

[42] Maria H; Marine bioactive compounds: sources, characterization, and applications. Springer, 2012: 238p.

[43] Bhatnagar I and Kim SK; Immense essence of excellence: marine microbial bioactive compounds.Marine drugs, 2010, 8(10): 2673-2701.

[44] Elga Š and al.; Sea Buckthorn Vegetative Parts - A Good Source of Bioactive Compounds, Proceedings of the Latvian Academy of Sciences. Section B. Natural, Exact, and Applied Sciences, 2013, 2(67): 101-108.

[45] Debbab A et al.; Bioactive Compounds from Marine Bacteria and Fungi. MicrobialBiotechnology, 2010, 3(5): 544-563.

[46] CorradoT; Bioactive compounds from natural sources: isolation, characterization and biological properties.London; New York: Taylor \& Francis, 2001: 693p.

[47] CorradoT; Bioactive compounds from natural sources: natural products as lead compounds in drug discovery, Boca Raton: CRC Press (2nd Ed), 2012: 622p.

[48] BeritSP;Highlights through the history of plant medicine. In: Bioactive compounds in plants - benefits and risks for man and animals. Oslo: The Norwegian Academy of Science and Letters, 2010: 23.

[49] Arseniyadis S and CossyJ; Modern Tools for the Synthesis of Complex Bioactive Molecules (1st Ed), John Wiley \& Sons, 2012: $581 p$

[50] JelenP; Book Review: Bioactive Components in Milk and Dairy Products. International Dairy Journal, 2010, 20(8): 560 .

[51] Goldberg G; Plants: Diet and Health. BNF (British Nutrition Foundation). Wiley \& Sons (London), 2003: 2-4.

[52] Biesalski HK et al.; Bioactive compounds: Safety and efficacy. Nutrition, 2009, 25(11-12): 1206-1211.

[53] Bhatnagar A et al.; A review on recent advances in Coumarin derivatives with their multidisciplinary actions. Der Pharmacia Lettre, 2010, 2(4): 297-306.

[54] Mehdi K et al.; Coumarin-Based Bioactive Compounds:
Facile Synthesis and Biological Evaluation of Coumarin-Fused 1,4-Thiazepines. Chemical Biology \& Drug Design, 2011, 78: 580-586.

[55] Sadia R et al.; Synthesis and in-vitro antimicrobial screening of 3-cinnamoyl coumarin and 3-[3-(1H-indol-2-yl)-3-aryl-propanoyl]-2H-chromen-2-ones. Iranian Journal of Catalysis, 2013, 3(3): 171-176.

[56] Borges F et al.; Simple Coumarins and Analogues in Medicinal Chemistry: Occurrence, Synthesis and Biological Activity. Current Medicinal Chemistry, 2005, 12(8): 887-916.

[57] Kinza A et al.; Synthesis and applications of coumarin. Pakistan Journal of Pharmaceutical Sciences. 2010, 23(4): 449-454.

[58] Nie $J$ et al.; Simultaneous determination of 6-methylcoumarin and 7-methoxycoumarin in cosmetics using three-dimensional excitation-emission matrix fluorescence coupled with second-order calibration methods. Talanta, 2008, 75:1260-1268.

[59] Konstantina $\mathrm{CF}$ et al.; Natural and Synthetic Coumarin Derivatives with Anti-Inflammatory/Antioxidant Activities. Current Pharmaceutical Design, 2004, 10(30): 3813-3833.

[60] Liu RH; Health-promoting components of fruits and vegetables in the diet. Advances in Nutrition, 2013, 4(3): 384 S-392S

[61] Walzem RL et al.; Whey components: millennia of evolution create functionalities for mammalian nutrition: what we know and what we may be overlooking. Critical Reviews in Food Science and Nutrition, 2002, 42: 353-375.

[62] Niels PM et al.; Bioactive peptides and proteins from foods: indication for health effects, European Journal of Nutrition, 2008, 47:171-182.

[63] Hernández-Ledesma $B$ et al.; $\beta$-Lactoglobulin as source of bioactive peptides. Amino Acids, 2008, 35(2): 257-265.

[64] Rocio CV et al.; Minor components of pulses and their potential impact on human health. Food Research International, 2010, 43(2): 461-482.

[65] Spatafora C and TringaliC; Valorization of Vegetable Waste: Identification of Bioactive Compounds and Their Chemo-Enzymatic Optimization. The Open Agriculture Journal, 2012, 6: 9-16.

[66] Barbosa-Pereira L et al. ; Fractionation and Purification of Bioactive Compounds Obtained from a Brewery Waste Stream. BioMed Research International, 2013: 1-11.

[67] Gui-Fang D, Potential of Fruit Wastes as Natural Resources of Bioactive Compounds. International Journal of Molecular Sciences, 2012; 13(7): 8308-8323. 